\title{
DESIGN AND IN VITRO CHARACTERIZATION OF MUCOADHESIVE BUCCAL PATCHES OF DULOXETINE HYDROCHLORIDE
}

\author{
HIMABINDU PEDDAPALLI ${ }^{1,2}$, KRISHNA MOHAN CHINNALA ${ }^{2}$, NAGARAJ BANALA ${ }^{*}$ \\ 1Department of Pharmaceutics, University College of Pharmaceutical Sciences, Kakatiya University, Warangal, Telangana, India 506009, \\ 2Department of Pharmaceutics, School of Pharmacy, Nalla Narasimha Reddy Education Society's Group of Institutions, Hyderabad, \\ Telangana, India 500088 \\ Email: bnrpharmacy@gmail.com \\ Received: 30 Jun 2016 Revised and Accepted: 07 Dec 2016
}

\begin{abstract}
Objective: Buccal mucosa is a potential site for the delivery of drugs to the systemic circulation, a drug administered through the buccal mucosa enters directly to the systemic circulation, thereby which bypass the drug from the firstpass hepatic metabolism and adverse gastrointestinal effect. Duloxetine hydrochloride (DLX HCL) is a selective serotonin and noradrenaline reuptake inhibitor (SSNRI). It is used in the treatment of depression, diabetic peripheral neuropathic pain and in moderate to severe stress urinary incontinence in women.

However, it undergoes extensive first-pass metabolism, and it is susceptible to undergo degradation in the acidic environment of the stomach, which results in the poor bioavailability. The objective of the present investigation is to design and evaluate the mucoadhesive buccal patches of
\end{abstract} DLX HCL with a goal of to increase the bioavailability and improve the patient compliance.

Methods: Mucoadhesive buccal patches were prepared by solvent casting technique using mucoadhesive polymers. The patches were evaluated for weight variation, thickness, surface $\mathrm{pH}$, folding endurance, moisture absorption, drug content uniformity, in vitro drug release, mechanical properties and ex vivo permeation studies.

Results: The results of the optimised buccal patch F4 indicate that the mucoadhesive buccal patches of duloxetine hydrochloride may be a good choice for improving the bioavailability by bypassing the extensive first pass metabolism and acid degradation in the stomach.

Conclusion: Duloxetine hydrochloride can be delivered through the buccal route of drug administration through the buccal patches.

Keywords: Mucoadhesive buccal patches, Duloxetine hydrochloride, Ex vivo permeation studies

(C) 2017 The Authors. Published by Innovare Academic Sciences Pvt Ltd. This is an open access article under the CC BY license (http://creativecommons.org/licenses/by/4. 0/) DOI: http://dx.doi.org/10.22159/ijpps.2017v9i2.13793

\section{INTRODUCTION}

Over the decades, peroral delivery has been the popular route of administration for the majority of therapeutic agents targeting systemic delivery. Oral administration generally leads to 'Transmucosal' absorption in the gastrointestinal tract; however, this enteral route of delivery subjects compound to extensive presystemic elimination, which may include gastrointestinal degradation, metabolism, or first-pass clearance via the liver, which leads to lack of significant correlation between membrane permeability, absorption, and bioavailability [1]. This first pass hepatic metabolism has often resulted in low systemic bioavailability, short duration of therapeutic activity, the formation of inactive agents and, at times, toxic metabolites [2].

Difficulties associated with invasiveness of parenteral delivery and poor oral availability provided the impetus for exploring alternative routes for the delivery of such drugs. Consequently, other absorptive mucosae are considered as potential sites for drug administration. Transmucosal routes of drug delivery (i.e., the mucosal linings of the nasal, rectal, vaginal, ocular, and oral cavity) offer attractive alternative routes for administration of drugs and may avoid the significant drawbacks of peroral and parenteral administration for systemic effect. The oral cavity, however, is a highly accepted route for both local and systemic drug delivery [3]. Buccal mucosa is a potential site for the delivery of drugs to the systemic circulation, a drug administered through the buccal mucosa enters directly to the systemic circulation through the internal jugular vein, thereby which bypass the drug from the first-pass hepatic metabolism and adverse gastrointestinal effect $[4,5]$. The buccal route of administration also offers a number of advantages [6], like good accessibility, the robustness of the epithelium, relatively permeable mucosa with a rich blood supply, termination of therapy at any time, comparatively less susceptibility to enzymatic activity and useful for paediatric and geriatric patients. Hence, adhesive mucosal dosage forms were prepared for oral delivery, in the form of adhesive tablets [7, 8], adhesive gels, buccal ointments $[9,10]$, and adhesive patches [11].

Duloxetine hydrochloride is a dual serotonin and norepinephrine reuptake inhibitor (SNRI). It used in the treatment of depression, diabetic peripheral neuropathic pain and in moderate to severe stress urinary incontinence in women. A daily dose of Duloxetine hydrochloride is $20 \mathrm{mg}$ or less is preferable. Hence, it can be conveniently loaded onto a patch. The molecular weight of drug is $330 \mathrm{~g} / \mathrm{mol}$. So the drug gets easily absorbed through buccal route. Duloxetine hydrochloride has excellent lipophilicity (Log p 4.2).

Oral administration of this drug has a lower bioavailability of about $42 \%$ only (18\%-71.2\%) because it undergoes extensive first pass metabolism by the cytochrome $\mathrm{P}_{450}$ isoenzymes CYP1A2 and CYP2D6 in the liver to numerous metabolites. The major biotransformation pathways for duloxetine involve oxidation of the naphthyl ring followed by conjugation and further oxidation. So its bioavailability may be significantly improved if delivered through the buccal route. Its biological half-life is $6.4 \mathrm{~h}$ will be good candidates for buccal drug delivery system. It was susceptible to undergo degradation in the acidic environment of the stomach (acid sensitive) will be assured protection in the buccal dosage form. Based upon the above reasons, it was considered as a potential alternative to formulate buccal drug delivery system for delivery of duloxetine hydrochloride, which can improves its bioavailability by avoiding hepatic metabolism and GIT degradation using suitable mucoadhesive polymers. The aim of the investigation of the present study was to develop new mucoadhesive buccal patches of duloxetine hydrochloride. 


\section{MATERIALS AND METHODS}

\section{Materials}

Duloxetine hydrochloride was a kind gift sample from Medreich Ltd, (Bangalore, India). Hydroxypropyl methylcellulose E 15 and Eudragit RL 100 were gift samples from Dr. Reddy's Laboratories (Hyderabad, India). Dichloromethane AR, methanol HPLC and AR were procured from Merck Ltd., (Mumbai, India). Propylene glycol was purchased from Fine chemicals, (Chandigarh, India). Phenol red was purchased from Hi-Media Laboratories Pvt. Ltd., (Mumbai, India).

\section{Methods}

\section{Preformulation studies}

\section{Solubility studies}

The solubility of DLX HCL in pH 6.6 phosphate buffer, distilled water and $7.4 \mathrm{pH}$ phosphate buffer was determined by phase equilibrium method. An excess amount of drug was taken into $50-\mathrm{ml}$ conical flasks containing $20 \mathrm{ml}$ of $\mathrm{pH} 6.6$ phosphate buffer, distilled water and $7.4 \mathrm{pH}$ phosphate buffer. These flasks were closed with aluminium foil and placed on a rotary shaker at room temperature for agitation for about 48hours. After $24 \mathrm{~h}$, the solution was filtered through a $0.2-\mu \mathrm{m}$ Whatman filter paper; the filtrate was collected, and the amount of drug solubilized was then estimated by measuring the absorbance at $290 \mathrm{~nm}$ using a UV spectrophotometer [12] (Elico Pvt Ltd, Hyderabad). The studies were repeated in triplicate $(n=3)$, and the mean was calculated.

\section{Drug excipients compatibility studies}

\section{Fourier transform infrared (FTIR) spectroscopy}

The Fourier transform infrared (FTIR) spectra for the samples were obtained using $\mathrm{KBr}$ disk method by FTIR spectrophotometer (BX I, Perkin Elmer, USA). Pure drug DLX, a Physical mixture of DLX and HPMC E15, a Physical mixture of DLX and Eudragit RL 100 and a Physical mixture of DLX, HPMC E15 and Eudragit RL100 were prepared and subjected to FTIR study. About 2-3 mg of sample was mixed with dried potassium bromide of equal weight and compressed to form a $\mathrm{KBr}$ disk. The samples were scanned from 400 to $4000 \mathrm{~cm}^{-1}$ spectral region with a resolution of $4 \mathrm{~cm}^{-1}$ as shown in the fig. 2.

\section{Ex vivo drug permeation studies through porcine buccal mucosa}

The aim of this study was to investigate the permeability of buccal mucosa to duloxetine hydrochloride. It is based on the generally accepted hypothesis that the epithelium is the rate-limiting barrier in buccal absorption [13]. The oral mucosa of pigs resembles that of humans more closely than any other animal in terms of structure and composition [14] and therefore porcine buccal mucosa was selected for drug permeation studies.

\section{Tissue preparation (Isolation)}

Porcine buccal tissue was taken from a local slaughterhouse. It was collected within $10 \mathrm{~min}$ after the slaughter of the pig and tissue was stored in Krebs buffer solution [15, 16]. It was transported immediately to the laboratory and was used within $2 \mathrm{~h}$ of isolation of buccal tissue.

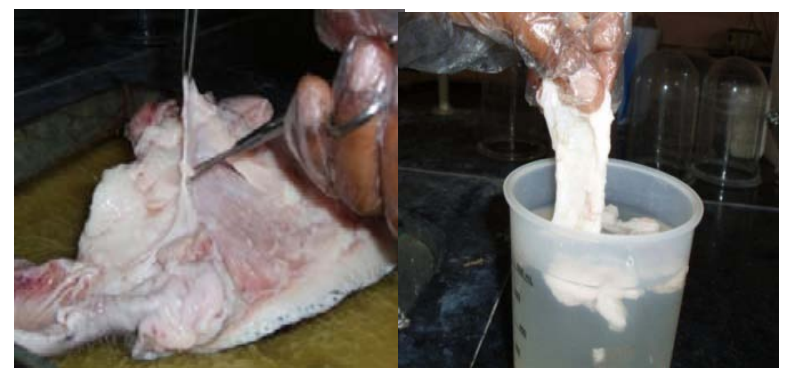

Fig. 1: Isolation of porcine buccal membrane by surgical technique
The tissue was rinsed thoroughly using phosphate buffer saline to remove any adherent material. The buccal epithelium was carefully separated from the underlying connective tissue with surgical technique, and then the remaining buccal mucosa was carefully trimmed with the help of surgical scissors to a uniform thickness [17]. Sufficient care was taken to prevent any damage to the buccal epithelium. Finally, the membrane was allowed to equilibrate for approximately one hour in receptor buffer to regain the lost elasticity.

\section{Procedure}

Ex vivo permeation study of duloxetine hydrochloride was performed through the porcine buccal mucosa using Franz diffusion cell [18]. The isolated buccal epithelium was carefully mounted between the two compartments of a Franz diffusion cell with an internal diameter of 2.1 $\mathrm{cm}\left(3.46 \mathrm{~cm}^{2}\right.$ area $)$ and the membrane was allowed to equilibrate for approximately one hour. After the buccal membrane was equilibrated for one hour with $\mathrm{pH} 7.4$ phosphate buffer solution between both the chambers, the receiver compartment was filled with $25 \mathrm{ml}$ fresh phosphate buffer solution ( $\mathrm{pH} 7.4$ ), and the donor compartment was charged with $4 \mathrm{ml}(1 \mathrm{mg} / \mathrm{ml})$ of drug solution. The donor compartment also contained phenol red at a concentration of $20 \mu \mathrm{g} / \mathrm{ml}$. This is because phenol red acts as a non-absorbable marker compound and is not expected to permeate through the porcine buccal membrane $[19,20]$. The absence of phenol red in the receiver compartment indicates the intactness of the buccal membrane. The entire setup was placed over magnetic stirrer at $50 \mathrm{rpm}$ and temperature was maintained at about $37^{\circ} \mathrm{C}$. The $2 \mathrm{ml}$ of samples were collected at predetermined time intervals $0.5,1.0,1.5,2.0,3.0,4.0,5.0$, and $6.0 \mathrm{hr}$ from receptor compartment and replaced with an equal volume of fresh buffer solution and stored under refrigerated conditions till the analysis was carried out. All the experiments were performed in triplicates. Finally, the amount of drug permeated through the buccal mucosa was determined by measuring the absorbance at $290 \mathrm{~nm}$ using a UV-Visible spectrophotometer.

\section{Calculation of Flux}

The cumulative amount of drug permeated, $Q$, through a unit surface area, $\mathrm{A}$, of a barrier was plotted against time, $\mathrm{t}$

The flux (J) was calculated by using the following equation

$$
\begin{aligned}
& \mathrm{J}=(\mathrm{dQ} / \mathrm{dt}) / \mathrm{A} \\
& \mathrm{P}=(\mathrm{dQ} / \mathrm{dt}) / \Delta \mathrm{CA}
\end{aligned}
$$

Where J was Flux (mg. hrs-1 $\mathrm{cm}^{-2}$ ); $\mathrm{P}$ is permeability coefficient $(\mathrm{cm} / \mathrm{h}) ; \mathrm{dQ} / \mathrm{dt}$ was the slope obtained from the steady state portion of the curve; $\Delta \mathrm{C}$, the concentration difference across the mucosa and A the area of diffusion $\left(\mathrm{cm}^{2}\right)$.

The target flux was calculated using following equation

$$
\mathrm{J}_{\text {Target }}=\frac{\text { Css CLT BW }}{\mathrm{A}}
$$

Where, BW is the standard human body weight of $60 \mathrm{~kg}$; $\mathrm{C}_{\mathrm{SS}}$, the duloxetine hydrochloride concentration at the therapeutic level, $\mathrm{CL}_{\mathrm{T}}$, the total body clearance and $\mathrm{A}$ the area of diffusion $\left(\mathrm{cm}^{2}\right)$.

\section{Formulation development}

Preparation of mucoadhesive buccal patches by solvent casting technique

Buccal patches were prepared using solvent casting technique [21] with hydroxypropyl methylcellulose (HPMC E15) and Eudragit RL100 as a mucoadhesive polymer, dichloromethane: methanol (1:1) as a solvent system and propylene glycol (PG) as plasticiser $[22,23]$. Weighed quantities of HPMC E15 and Eudragit RL100 as shown in the table were taken in a boiling tube. To this, $25 \mathrm{ml}$ of a solvent mixture of dichloromethane: methanol (1:1) was added and vortexed. Sufficient care was taken to prevent the formation of lumps. The boiling tube was set-aside for $6 \mathrm{~h}$ to allow the polymer to swell. After swelling, measured quantity of propylene glycol was added to this mixture and vortexed. Finally weighed the quantity of duloxetine hydrochloride was dissolved in $5 \mathrm{ml}$ of the solvent 
mixture, added to the polymer solution and mixed well. It was setaside for some time to remove any entrapped air. The gel was then transferred into a previously cleaned anumbra petri plate. Drying of these patches was carried out in an oven placed over a horizontal surface, with the temperature being maintained at $40{ }^{\circ} \mathrm{C}$ for about $24 \mathrm{~h}$, till a flexible film was formed.
The developed buccal patches were circular in shape with a 0.2 to $0.4 \mathrm{~mm}$ thickness and containing $10 \mathrm{mg}$ of drug per patch. These developed patches were removed carefully and cut into small square shaped pieces, each patch having an area $2.89 \mathrm{~cm}^{2}$ and containing 10 $\mathrm{mg}$ of drug per patch. They were packed in aluminium foil till the evaluation tests were performed.

Table 1: The composition of the duloxetine hydrochloride buccal patches

\begin{tabular}{llllll}
\hline Code & $\begin{array}{l}\text { Duloxetine hydrochloride } \\
\text { (mg) }\end{array}$ & $\begin{array}{l}\text { HPMC E15 } \\
\text { (mg) }\end{array}$ & $\begin{array}{l}\text { Eudragit RL100 } \\
\text { (mg) }\end{array}$ & $\begin{array}{l}\text { Propylene glycol } \\
\text { ( } \boldsymbol{\mu l} \text { ) }\end{array}$ & $\begin{array}{l}\text { Solvent system } \\
\text { Dichloromethane: methanol 1:1 (ml) }\end{array}$ \\
\hline$E_{1}$ & 220 & 1760 & - & 264 & 30 \\
$E_{2}$ & 220 & 2200 & - & 330 & 30 \\
$F_{1}$ & 220 & 880 & 220 & 165 & 30 \\
F2 & 220 & 1100 & 220 & 198 & 30 \\
F3 & 220 & 1320 & 220 & 231 & 30 \\
F4 & 220 & 1540 & 220 & 264 & 30 \\
F5 & 220 & 1980 & 220 & 330 & 30 \\
\hline
\end{tabular}

Formulations $\mathrm{E}_{1}$ and $\mathrm{E}_{2}$ were prepared using HPMC $\mathrm{E} 15$ with the drug: polymer ratio 1:8, 1:10 respectively. Formulation $\mathrm{F}_{1}$ to $\mathrm{F}_{5}$ were prepared using HPMC E 15 and Eudragit RL100 with the drug: polymer ratio 1:5, 1:6, 1:7, 1:8, and 1:10 respectively. The amount of Eudragit RL 100 was kept constant. Each patch $\left(2.89 \mathrm{~cm}^{2}\right)$ contained $10 \mathrm{mg}$ of DLX. $15 \% \mathrm{v} / \mathrm{w}$ of propylene glycol to the total polymer weight was incorporated as a plasticiser.

\section{Evaluation of the developed buccal patches}

\section{Weight variation test}

Six patches each equivalent to $2.89 \mathrm{~cm}^{2}$ area was cut from each plate and their weight was measured individually using Shimadzu digital balance and the average weight was calculated. The mean \pm SD values were calculated for all the formulated patches.

\section{Thickness variation test}

The thickness of the patches was measured at six different points of the patch by digital gauge (Mitutoyo, Japan). The mean \pm SD values were calculated for all the formulations.

\section{Folding endurance}

Folding endurance of the patches was determined by repeatedly folding one patch at the same place till it broke or folded up to 200 times manually, which was considered satisfactory to reveal good patch properties. The number of times of patch could be folded at the same place without breaking gave the value of the folding endurance of patch [24].

\section{Surface pH of films}

The method adopted by Bottenberg et al. [25], was used to determine the surface $\mathrm{pH}$ of the patches. A combined glass electrode was used for this purpose. The bioadhesive buccal patch was made in contact with 1 $\mathrm{ml}$ of distilled water and allowed to swell for 1-2 $\mathrm{h}$ at room temperature. The surface $\mathrm{pH}$ of the patches was measured by bringing the $\mathrm{pH}$ meter electrode in contact with the surface of the patch and allowing it to equilibrate for 1 minute.

\section{Assay of the patches}

The formulated patches were assayed for drug content in each case. Three patches from each formulation equivalent to $2.89 \mathrm{~cm}^{2}$ area were assayed for content of drug. Each formulation was casted in triplicate and one patch from each was taken and assayed for content of drug.

\section{Procedure}

Patches from each formulation series were taken, and each patch was cut into small pieces. The pieces were taken into a $100 \mathrm{ml}$ of conical flask, allowed to dissolve in $100 \mathrm{ml}$ of $\mathrm{pH} 6.6$ phosphate buffer. The solution was filtered through $0.45 \mu \mathrm{m}$ filter paper and diluted appropriately with phosphate buffer $\mathrm{pH} 6.6$ and the drug content was estimated using UV-Visible spectrophotometer (Elico, India) at $290 \mathrm{~nm}$.

\section{In vitro drug release studies}

Drug release from the bioadhesive buccal patch was studied by using the USP type II dissolution test apparatus. The dissolution medium consisted of $500 \mathrm{ml}$ of phosphate buffer $\mathrm{pH}$ 6.6. The experiment was performed at $37 \pm 0.2{ }^{\circ} \mathrm{C}$, with a rotation speed of $50 \mathrm{rpm}$. Patches of the desired size were cut, and since the patches were meant to release the drug from only one side, therefore an impermeable backing membrane was placed on one side of the patch. The dissolution assembly was prepared by adhering the patch onto a $2 \times 2 \mathrm{~cm}$ glass slide with a solution of cyanoacrylate adhesive [26]. It was then placed in dissolution apparatus. Samples of $5 \mathrm{ml}$ were collected at predetermined time intervals: 0.5, 1.0, 1.5, 2.0, 3.0, 4.0, 5.0 and $6.0 \mathrm{hr}$ and the equivalent amount were replaced by fresh medium. The samples were filtered through a $0.2-\mu \mathrm{m}$ Whatman filter paper and analysed using UV-Visible spectrophotometer at $290 \mathrm{~nm}$.

\section{Moisture absorption studies}

The moisture absorption studies give an indication about the relative moisture absorption capacities of polymers and an idea whether the formulation maintains its integrity after absorption of moisture. This test was performed in accordance with the procedure reported earlier [27]. $5 \% \mathrm{w} / \mathrm{v}$ agar in distilled water, in hot condition, was transferred into Petri plates and it was allowed to solidify. Then six patches from each formulation were selected and weighed. They were placed on the surface of the agar and incubated at $37{ }^{\circ} \mathrm{C}$ for two hours in an incubator. The patches were removed and weighed again. The percentage of moisture absorbed can be calculated using the following formula.

$$
\% \text { Moisture absorbed }=\frac{\text { Final weight }- \text { Initial weight }}{\text { Initial weight }} \times 100
$$

\section{Measurement of mechanical properties}

Mechanical properties of the patches were evaluated using a microprocessor based advanced force gauze equipped with a motorised test stand (Ultra Test, Mecmesin, West Sussex, UK) equipped with a $25 \mathrm{~kg}$ load cell. Film strip with the dimensions $60 \mathrm{x}$ $10 \mathrm{~mm}$ and free from air bubbles or physical imperfections, were held between two clamps positioned at a distance of $3 \mathrm{~cm}$. Cardboard was attached on the surface of the clamp to prevent the film from being cut by the grooves of the clamp. During measurement, the strips were pulled by the top clamp at a rate of $2.0 \mathrm{~mm} / \mathrm{s}$ to a distance till the film broke. The force and elongation were measured when the films were broken. Results from film samples, which were broken at the end and not between the clamps were not included in observations. Measurements were run in six replicates for each formulation. The following equations were used to calculate the mechanical properties of the films. Results are presented in table 4 .

Tensile strength $\left(\mathrm{kg} \cdot \mathrm{mm}^{-2}\right)=\frac{\text { Force at break }(\mathrm{kg})}{\text { Initial cross sectional area of the sample }\left(\mathrm{mm}^{2}\right)}$ 
Elongation at break $\left(\% \mathrm{~mm}^{-2}\right)=\frac{\text { Increase in length }(\mathrm{mm})}{\text { Original length } \mathrm{x} \text { Cross sectional area }\left(\mathrm{mm}^{2}\right)} \times 100$

\section{In vitro bioadhesion measurement}

The adhesive properties of patches containing duloxetine was determined using a microprocessor based advanced force gauze equipment with a porcine buccal membrane as a model tissue under simulated buccal conditions. A microprocessor based advanced force gauge equipped with a motorised test stand (Ultra Test, Mecmesin, West sussex, UK), equipped with a $5 \mathrm{~kg}$ load cell was employed to determine the bioadhesion. The porcine buccal membrane was secured tightly to a circular stainless steel adaptor (diameter $2.2 \mathrm{~cm}$ ) provided with the equipment as an accessory. This was fixed to advanced force gauze. The buccal patch to be tested was placed over another cylindrical stainless steel adaptor of similar diameter and mounted onto the platform of the motorised test stand. Buccal patch with the backing membrane was adhered onto it using a solution of cyanoacrylate adhesive. During measurement, $100 \mu \mathrm{l}$ of $1 \%$ mucin solution (crude mucin) was used to moisten the porcine buccal membrane. The upper support was lowered at a speed of $0.5 \mathrm{~mm} / \mathrm{s}$ until contact was made with the tissue at a predetermined force of $0.5 \mathrm{~N}$ for a contact time of $180 \mathrm{sec}$. At the end of the contact time, upper support was withdrawn at a speed of $0.5 \mathrm{~mm} / \mathrm{s}$ to detach the membrane from the patch. Data collection and calculations were performed using the data plot software package of the instrument. Two parameters, namely the work of adhesion and peak detachment force were used to study the buccal adhesiveness of patches [28]. The work of adhesion was determined from the area under force distance curve while the peak detachment force was the maximum force required to detach the film from the buccal tissue.

\section{Stability of buccal patches}

Stability studies of buccal patches were performed for optimised formulation (F4) in natural human saliva. The saliva was collected from humans (aged 22-26) and filtered through Whatman $(0.2 \mu \mathrm{m})$ filter paper. Buccal patches were placed in separate petri dishes containing $5 \mathrm{ml}$ of human saliva and placed in a temperaturecontrolled oven for $6 \mathrm{~h}$ at $37 \pm 0.2^{\circ} \mathrm{C}$. At regular time intervals $(0,2,4$, and $6 \mathrm{~h}$ ), the buccal patches were examined for change in color, surface area, and integrity [28]. The experiments were repeated in triplicate $(n=3)$ in a similar manner.

Ex vivo permeation of duloxetine hydrochloride through porcine buccal membrane from optimised buccal patch

Ex vivo permeation of duloxetine from the buccal patch for the optimised formulation (F4) through the porcine buccal membrane was studied. The buccal membrane was isolated as described in tissue preparation section. The membrane was mounted over a
Franz diffusion cell whose internal diameter $2.1 \mathrm{~cm}$. The buccal patch was sandwiched between the buccal mucosa and the dialysis membrane, so as to secure the patch tightly from getting dislodged from the buccal membrane. $25 \mathrm{ml}$ of phosphate buffer $\mathrm{pH} 7.4$ was placed in the receptor compartment. The entire set up was placed over magnetic stirrer and the temperature was maintained at $37^{\circ} \mathrm{C}$. Samples of $2 \mathrm{ml}$ were collected at predetermined time points from receptor compartment and replaced with an equal volume of buffer. The amount of drug permeated from optimised formulation through the buccal mucosa was then determined by measuring the absorbance at $290 \mathrm{~nm}$ using a UV-Visible spectrophotometer.

In vitro-ex vivo correlation between cumulative \% drug released in vitro and \% drug permeated ex vivo of optimised duloxetine hydrochloride buccal patch

A possible in vitro-ex vivo correlation was performed for \% drug released in vitro and \% drug permeated ex vivo for optimised formulation [24]. Results were presented in fig. 6.

\section{RESULTS AND DISCUSSION}

\section{Solubility studies}

Solubility studies of duloxetine hydrochloride were carried out. The studies were repeated in triplicate $(\mathrm{n}=3)$, and mean was calculated. The solubility of duloxetine hydrochloride was found to be 15.56 , 14.83 and $14.24 \mathrm{mg} / \mathrm{ml}$ in $6.6 \mathrm{pH}$ phosphate buffer, distilled water and $7.4 \mathrm{pH}$ phosphate buffers respectively. Maximum solubility in $6.6 \mathrm{pH}$ phosphate buffer was noticed, and order of solubility is 6.6 $\mathrm{pH}$ phosphate buffer $>$ distilled water $>7.4 \mathrm{pH}$ phosphate buffer.

\section{Drug-excipient compatibility studies}

\section{Fourier transform infrared (FTIR) spectroscopy studies}

The potential chemical interaction between drug and polymer may change the therapeutic efficacy of the drug. To investigate the possibility of any chemical interaction between drug and polymers used in the preparation of patches FTIR spectroscopic studies were carried out; samples were analysed over the range 400-4000 $\mathrm{cm}^{-1}$. Fig. shows FTIR spectrum of pure drug DLX, a physical mixture of DLX and HPMC E15, a physical mixture of DLX and Eudragit RL 100, and physical mixture of DLX, HPMC E15 and Eudragit RL 100.

The FTIR spectrum of DLX showed principal bands at $1490 \mathrm{~cm}^{-1}$ for thiophene ring, $3000-3010 \mathrm{~cm}^{-1}$ for aromatic alkene proton $(\mathrm{C}=\mathrm{C}-\mathrm{H})$, at $1400-1600 \mathrm{~cm}^{-1}$ for aromatic alkene, $1000-1300 \mathrm{~cm}^{-1}$ for ether C-O and at $1080-1360 \mathrm{~cm}^{-1}$ for C-N bond. These FTIR bands of the drug remain intact in both the spectra of the drug and physical mixture, the illustrating absence of interaction between drug and mucoadhesive polymers used.

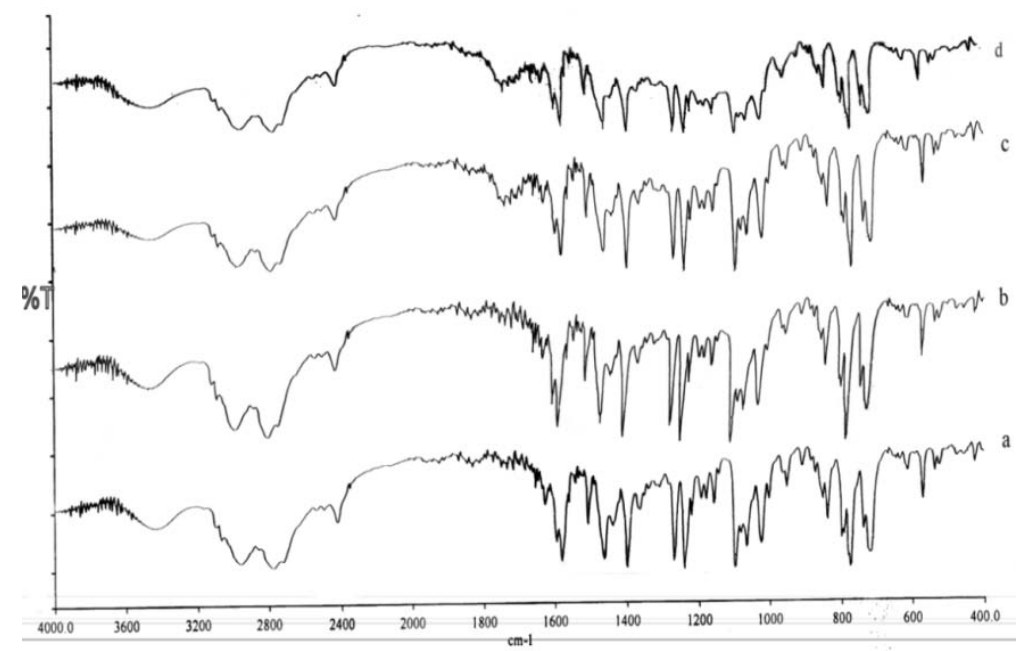

Fig. 2: FTIR spectrum of DLX (a), Physical mixture of DLX and HPMC E15 (b), Physical mixture of DLX and Eudragit RL 100 (c), Physical mixture of DLX, HPMC E15 and Eudragit RL 100 (d) 


\section{Ex-Vivo drug permeation studies through porcine buccal mucosa}

Porcine buccal mucosa had been the most frequently chosen model tissue for ex vivo permeation studies because of its similarity to human tissue in terms of thickness and is available in large quantities from the slaughterhouse. No detectable levels of phenol red (marker compound) were found in the receiver compartment indicates the intactness of buccal membrane, whereas DLX could permeate freely. The cumulative percentage amount of DLX permeated through the buccal membrane in first two hrs was $46.43 \%$ and $81.25 \%$ in $6 \mathrm{~h}$ clearly indicates that the penetration of the drug through the porcine buccal epithelium was initially rapid and followed by slow penetration rate. The flux was calculated to be $0.139 \pm 0.168 \mathrm{mg} \mathrm{hrs}^{-1} \mathrm{~cm}^{-2}$ (Target flux $0.143 \mathrm{mg} \mathrm{hrs}^{-1} \mathrm{~cm}^{-2}$ ). The cumulative percentage amount of duloxetine hydrochloride that had penetrated through the buccal epithelium is shown in the fig. 3.

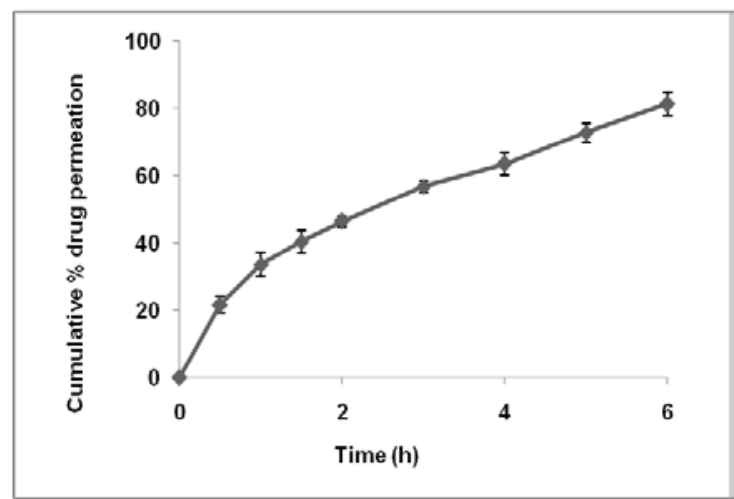

Fig. 3: Ex vivo permeation of duloxetine hydrochloride through porcine buccal mucosa

\section{Formulation of duloxetine hydrochloride buccal patches}

Preparation of patches by solvent casting technique

The single layered patches with a combination of polymers were prepared by solvent casting method by using the polymers HPMC E15 and Eudragit RL 100.

Primary trails were conducted to develop DLX buccal patches using HPMCE15 as a mucoadhesive polymer. The patch E1 (1:8) and E2 (1:10) released the drug much faster, about $100 \%$ release were achieved within $2 \mathrm{~h}$ and $3 \mathrm{~h}$ respectively. The buccal patches containing HPMC E15 and Eudragit RL100 were successfully prepared by solvent casting technique. These results are showed in fig. 4 at in vitro drug release studies. Patches with any imperfections, entrapped air, differences in weight were excluded from the further studies.

\section{Evaluation of the developed buccal patches}

\section{Weight, thickness and drug uniformity}

The prepared patches were smooth, elegant in appearance, uniform in weight, thickness, and drug content and showed no visible cracks and showing good folding endurance. Table 2 shows the important physicochemical parameters of Eudragit buccoadhesive patches of duloxetine hydrochloride. The weight of the patches ranged from $63 \pm 0.52$ to $117 \pm 0.86 \mathrm{mg}$ which indicates that weight of patches are increased with increase in polymer concentration in formulations. The thickness ranged from $211 \pm 0.06 \mu \mathrm{m}$ to $468 \pm 0.03 \mu \mathrm{m}$ and was found to be increased with increase in polymer concentration. The drug content in the buccal patches was uniform showed that the drug was dispersed uniformly throughout the patches. The drug content of buccal patches was found to be in the range of $95.5 \pm 0.6 \%$ to $99.8 \pm 0.6 \%$. The mean and standard deviations were calculated. All these parameters were within acceptable limits.

Table 2: Physicochemical parameters of mucoadhesive buccal patches of Duloxetine hydrochloride

\begin{tabular}{|c|c|c|c|c|c|}
\hline $\begin{array}{l}\text { Formulation } \\
\text { code }\end{array}$ & $\begin{array}{l}\text { Weight } \\
\text { variation (mg) }\end{array}$ & Thickness $(\mu \mathrm{m})$ & $\%$ drug content & Surface pH & $\begin{array}{l}\text { Folding } \\
\text { endurance }\end{array}$ \\
\hline F1 & $63 \pm 0.52$ & $211 \pm 0.06$ & $96.8 \pm 0.5$ & $6.83 \pm 0.55$ & $>200$ \\
\hline $\mathrm{F} 2$ & $75 \pm 0.61$ & $265 \pm 0.07$ & $97.6 \pm 0.8$ & $6.73 \pm 0.64$ & $>200$ \\
\hline F3 & $87 \pm 1.00$ & $368 \pm 0.04$ & $95.5 \pm 0.6$ & $6.68 \pm 0.68$ & $>200$ \\
\hline F4 & $94 \pm 0.76$ & $424 \pm 0.05$ & $98.4 \pm 0.7$ & $6.59 \pm 0.57$ & $>200$ \\
\hline F5 & $117 \pm 0.86$ & $468 \pm 0.03$ & $99.8 \pm 0.6$ & $7.12 \pm 0.34$ & $>200$ \\
\hline
\end{tabular}

Each value represents the mean $\pm \operatorname{SD}(n=3)$

\section{Surface pH study}

Surface $\mathrm{pH}$ of the patches was determined in order to investigate the possibility of any side effects, in vivo. As an acidic or alkaline $\mathrm{pH}$ may cause irritation to the buccal mucosa, it was our attempt to keep the surface $\mathrm{pH}$ as close to neutral as possible. The results showed that surface $\mathrm{pH}$ of all the patches ranged from $6.59 \pm 0.57$ to $7.12 \pm 0.34$ and found to be within an acceptable salivary pH (5.8 to 7.4). Hence, these patches may not cause any irritation to the buccal cavity.

\section{In vitro drug release studies}

The drug release profiles of duloxetine from buccal patches are shown in fig. 4.

In the case of formulations E1, E2 containing HPMC E15 alone in the ratio of 1:8, 1:10 (drug: polymer) showed about $98.88 \%$ and 99.83 $\%$ of drug release in $2 \mathrm{~h}$ and $3 \mathrm{~h}$ respectively. This because the polymer HPMC E 15 used was a low viscosity polymer and unlike the other grades of polymer like HPMC K4M, K15 or K100M and HPMC E 15 dissolves much faster and the drug was diffused from the patches onto the surface. Therefore to overcome the problem and to modify the drug release rate, a hydrophobic polymer Eudragit RL 100 was incorporated as a retarding agent.
In the case of F series formulations, F1, F2, F3 initially the drug release was rapid, more than $60 \%$ in $1.5 \mathrm{~h}$ and followed by slow release and showed about $96.28 \pm 3.14 \%, 94.52 \pm 2.16 \%$, $91.23 \pm 0.96 \%$ in $4 \mathrm{~h}$ respectively. The formulations F4 and F5 had shown $83.72 \pm 1.56 \%$ and $78.62 \pm 1.23 \%$ in $4 \mathrm{~h}$. There appeared no significant difference in the final percentage of drug release, which might be due to the fact that in all the formulations the drug dissolved completely in the dissolution medium. It is clear from the plots that the drug release was governed by polymer content.

No lag time was observed as the patch was directly exposed to the dissolution medium. An increase in the polymer content was associated with a decrease in drug release rates. This is because increasing the amount of polymer in the patches forms a waterswollen gel-like state that could substantially reduce the penetration of the dissolution medium into the patches and so the drug release was retarded.

The Formulation F4 was selected as optimised formulation based on these in vitro release studies which showed satisfactory drug release rates $98.76 \pm 2.14 \%$ in $6 \mathrm{~h}$. The selected optimised formulation $\mathrm{F} 4$ was used further for the evaluation of in vitro bioadhesive studies and ex vivo permeation studies through the porcine buccal mucosa. 


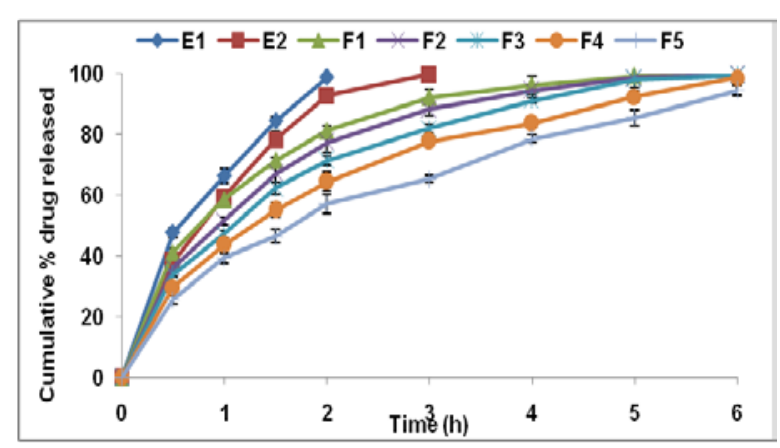

Fig. 4: In vitro drug release profiles of DLX from mucoadhesive buccal patches containing Eudragit RL100 and HPMC E15

\section{Mathematical modeling of dissolution profiles}

There are a number of kinetic models, which described the overall release of drug from the dosage forms. Data obtained from the in vitro release was fit into different equations and kinetic models to explain the release kinetics of duloxetine hydrochloride from these buccal patches. The kinetic models used were a zero-order equation, first order equation, Higuchi and Korsmeyer-Peppas model. Higuchi model is the most widely used model to describe drug release from pharmaceutical matrices system. The Peppas model is widely used, when the release mechanism is not well known and when more than one type of release is involved [29]. To find out the mechanism of drug release, drug release data were fitted in Korsmeyer-Peppas model [30].

$$
\mathrm{M}_{\mathrm{t}} / \mathrm{M}_{\infty}=\mathrm{Kt}^{\mathrm{n}}
$$

Where $M_{t} / M_{\infty}$ is a fraction of drug released at time $t$,

$\mathrm{K}$ is the release rate constant, and

$\mathrm{n}$ is the release exponent.

In this model, the value of $n$ characterises the type of release mechanism of the drug during the dissolution process. For nonFickian release, the value of $\mathrm{n}$ falls between 0.5 and 1 , while in the case of Fickian diffusion, $\mathrm{n}=0.5$; for zero order release (case II transport), $\mathrm{n}=1$; and for super case II transport, $\mathrm{n}$ is greater than 1 . To study the diffusional release mechanism, data obtained from in vitro drug release studies were plotted against log cumulative percentage drug release versus log time. The value of $n$ was estimated by linear regression of $\log \mathrm{M}_{\mathrm{t}} / \mathrm{M}_{\infty}$ versus $\log \mathrm{t}$. The $\mathrm{R}^{2}$ values and $\mathrm{n}$ values are presented in table 3 .

Table 3: Regression coefficient $\left(\mathrm{R}^{2}\right)$ values of duloxetine hydrochloride buccal patches for different kinetic models

\begin{tabular}{|c|c|c|c|c|c|}
\hline Formulation code & Zero-order $\mathbf{R}^{2}$ & First-order $\mathbf{R}^{2}$ & Higuchi R $^{2}$ & Peppas $\mathbf{R}^{2}$ & n value \\
\hline F1 & 0.872 & 0.916 & 0.931 & 0.895 & 0.434 \\
\hline $\mathrm{F} 2$ & 0.834 & 0.929 & 0.972 & 0.912 & 0.552 \\
\hline F3 & 0.873 & 0.779 & 0.951 & 0.879 & 0.538 \\
\hline $\mathrm{F} 4$ & 0.943 & 0.921 & 0.992 & 0.916 & 0.612 \\
\hline F5 & 0.965 & 0.957 & 0.993 & 0.924 & 0.625 \\
\hline
\end{tabular}

The optimised formulation (F4) followed Zero order release and Higuchi release kinetics because they showed high regression coefficient for Higuchi model and governed by a non-Fickian mechanism as evidenced from $\mathrm{n}$ value obtained from KorsmeyerPeppas equation. It was concluded that the release of drug from the films followed the diffusion controlled mechanism in all the formulations.

\section{Moisture absorption studies}

Moisture absorption studies evaluated the integrity of the formulation upon exposure to moisture. The results of moisture absorption studies were presented in the table. Percentage moisture absorbed values ranged from about $30.6 \pm 2.21$ to $81.2 \pm 2.04 \% \mathrm{w} / \mathrm{w}$. When the patches were placed without backing complete membrane swelling followed by erosion was observed indicating that the drug release mechanism involves swelling of the polymer initially followed by drug release from swollen matrix by diffusion.

\section{Mechanical properties of patches}

Ideal buccal patch, apart from good bioadhesive strength, should be flexible, elastic, and strong enough to withstand breakage due to stress caused during its residence in the mouth. The tensile strength (TS) and elongation at break (E/B) shows the strength and elasticity of the films. The results of the mechanical properties, i.e., tensile strength and elongation at break were presented in table 4 . Tensile strength increased with increase in the polymer content, but elongation at break values decreased with the increase in polymer content. The optimised formulation F4 exhibited Tensile strength of $15.30 \pm 2.14 \mathrm{~kg} / \mathrm{mm}^{2}$ and elongation at break of $54.5 \pm 3.04 \% \mathrm{~mm}^{-2}$.

\section{In vitro bio-adhesion studies}

In vitro bio-adhesion measurements were performed routinely for mucoadhesive dosage forms and the most commonly used technique for evaluation of buccal patches was the measurements of adhesive strength. Work of adhesion was calculated from the area under the force distances curve, a measure of work that must be done to remove a patch or film from the tissue. Peak detachment force is the maximum applied force at which the patch detaches from the tissue. For the optimised formulation $\mathrm{F} 4$, the peak detachment force and work of adhesion were found to be $3.12 \pm 0.32 \mathrm{~N}$ and $1.23 \pm 0.21 \mathrm{~mJ}($ mean $\pm S \mathrm{D} ; \mathrm{n}=3$ ) respectively. The work of adhesion and peak detachment force values was within the range for suitable bioadhesion as reported for various films.

Table 4: Percentage moisture absorbed and Mechanical properties of buccal patches

\begin{tabular}{|c|c|c|c|}
\hline Formulation code & \% moisture absorbed & Tensile strength $\left(\mathrm{kg} / \mathrm{mm}^{2}\right)$ & Elongation at break $\left(\% \mathrm{~mm}^{-2}\right)$ \\
\hline F1 & $30.6 \pm 2.21$ & $2.36 \pm 0.36$ & $91 \pm 3.82$ \\
\hline $\mathrm{F} 2$ & $37.2 \pm 5.29$ & $4.02 \pm 1.13$ & $83 \pm 2.38$ \\
\hline F3 & $51.35 \pm 3.61$ & $8.12 \pm 1.43$ & $71 \pm 4.36$ \\
\hline $\mathrm{F} 4$ & $69.4 \pm 1.46$ & $15.30 \pm 2.14$ & $54.5 \pm 3.04$ \\
\hline F5 & $81.2 \pm 2.04$ & $18.3 \pm 2.21$ & $46.2 \pm 2.27$ \\
\hline
\end{tabular}

Values are expressed as mean $\pm \mathrm{SD} ; \mathrm{n}=3$

\section{Stability of buccal patches}

Based on the above result, stability studies were conducted only for optimised formulation F4. From the stability studies, it was known that optimised formulation F4 had stability in human saliva; there was no change in the colour and integrity of the patches. Physical properties of the DLX HCL buccal patches such as thickness and diameter are slightly changed owing to swelling of the system in human saliva. The change in Surface area $\left(\mathrm{cm}^{2}\right)$ at $0,2,4$, and $6 \mathrm{~h}$ was found to be $0.2,1.05,1.26$, and $1.87 \mathrm{~cm}^{2}$, respectively. 
Ex vivo permeation of duloxetine hydrochloride through porcine buccal membrane from optimised buccal patch

The formulation $\mathrm{F} 4$ was selected as optimised formulation for the $e x$ vivo permeation studies due to its adequate in vitro drug release, its capacity to retain the structure in moisture absorption studies, and in vitro bio-adhesion studies. The results of drug permeation from buccal patches of Duloxetine hydrochloride through the porcine buccal mucosa reveal that drug was released from the formulation and permeated through the porcine buccal membrane and hence could possibly permeate through the human buccal membrane. The results indicated that the drug permeation was slow and steady and $72.81 \pm 2.86 \%$ of DLX could permeate through the buccal membrane from the optimised formulation in $6 \mathrm{~h}$ and the flux was calculated to be $0.167 \pm 0.18 \mathrm{mg} \mathrm{hrs}^{-1} \mathrm{~cm}^{-2}$ (Target flux $0.171 \mathrm{mg} \mathrm{hrs}^{-1} \mathrm{~cm}^{-2}$ ). The cumulative percentage amount of DLX that had penetrated through the buccal epithelium from buccal patch was shown in the fig. 6 .

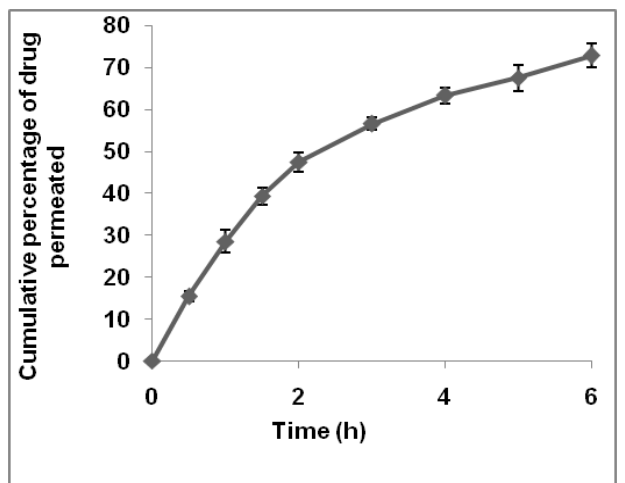

Fig. 5: Ex vivo permeation of Duloxetine hydrochloride from optimised formulation $F 4$ through porcine buccal mucosa, the values represented mean $\pm S D(n=3)$

In vitro-ex vivo correlation between cumulative \% drug released in vitro and \% drug permeated ex vivo of optimised DLX Buccal patch

Cumulative percentage of Duloxetine hydrochloride permeated through the porcine buccal membrane was correlated against cumulative percentage of drug released using in vitro release tests for optimised formulation F4. Fig. 6 shows the relationship between the percentage of Duloxetine hydrochloride released in vitro and percentage of drug permeated ex vivo. The straight line and the high correlation coefficient of 0.995 proved the good correlation between in vitro drug release and ex vivo drug permeation studies. Hence by considering the complete difference in the test conditions of in vitro and ex vivo release studies, the high correlation and coincidence of in vitro and ex vivo release profiles, it can be concluded that such a mucoadhesive patches could be a useful carrier in buccal drug delivery systems.

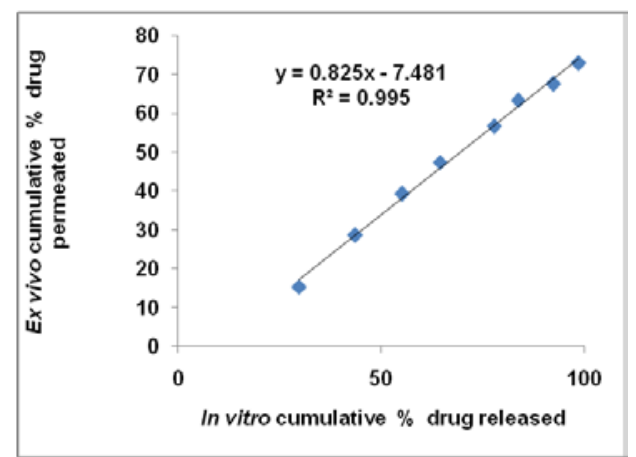

Fig. 6: In vitro-ex vivo correlation between cumulative \% drug released in vitro and \% drug permeated ex vivo of optimised DLX Buccal patch (F4)

\section{CONCLUSION}

Buccal delivery is an attractive alternative route for administration of drugs that has low bioavailability due to extensive first-pass metabolism. The following conclusion could be drawn from the results of various experiments. Duloxetine hydrochloride could permeate through the porcine buccal membrane as evidenced from the results of ex vivo drug permeation studies. FTIR studies concluded that there was no interaction between drug and excipients. The buccal patches of Duloxetine hydrochloride could be prepared by the solvent casting method with mucoadhesive polymers like HPMC E15 and Eudragit RL100. The prepared patches were smooth, elegant in appearance, uniform in weight, thickness, content uniformity and showed no visible cracks and showing good folding endurance. The Physicochemical properties of all the formulations were shown to be within limits. The surface $\mathrm{pH}$ of all the formulations was in an acceptable salivary $\mathrm{pH}$ (5.8 to 7.4). Hence, they do not cause any irritation to the buccal cavity. The optimised buccal patch F4 showed satisfactory drug release rates with the Higuchi model release profile. Buccal patches had shown good mechanical properties measured in terms of tensile strength and elongation at break values. Optimised buccal patches developed for DLX possess reasonable bio-adhesion measured in terms of peak detachment force and work of adhesion. From the stability studies, it has concluded that the buccal patches have maintained their integrity in the natural human saliva and exhibiting sufficient strength of the system throughout the experiment. Ex vivo permeation studies for optimised patches was conducted and shown satisfactory drug permeation. This could demonstrate that the optimised formulations could meet the target flux. Good in vitro ex vivo correlation for optimised buccal patch of Duloxetine hydrochloride demonstrates the validity of the release tests conducted. Hence, present study concludes that the Duloxetine hydrochloride could be delivered through the buccal route. Further work was recommended to support its efficacy claims by pharmacokinetic and pharmacodynamics studies in a human being.

\section{CONFLICT OF INTERESTS}

\section{Declared none}

\section{REFERENCES}

1. Harris D, Robinson JR. Drug delivery via the mucous membranes of the oral cavity. J Pharm Sci 1992;81:1-10.

2. Gandhi RB, Robinson JR. Oral cavity as a site for bioadhesive drug delivery. Adv Drug Delivery Rev 1994;13:43-74.

3. Shojaei AH, Zhuo SL, Li X. Transbuccal delivery of acyclovir (II): feasibility, system design, and in vitro permeation studies. J Pharm Sci 1998;1:66-73.

4. Senel S, Hincal AA, Squier CA. Effect of chitosan in enhancing drug delivery across buccal mucosa. Adv Chitin Sci, University Potsdam 2000;4:254-8.

5. Merkle HP, Anders R, Wermerskirchin A. In: Bioadhesive drug delivery systems, CRC Press: Boca Raton, Florida; 1990. p. 105.

6. Rojanasakul Y, Wang LY, Bhat M, Glover DD, Malanga CJ, Ma JKH. The transport barrier of epithelia; a comparative study on membrane permeability and charge selectivity in the rabbit. Pharm Res 1992;9:1029-34.

7. Davis SS, Daly PB, Kennerley JW, Frier M, Hardy JG, Wilson CG. Design and evaluation of sustained release formulations for oral and buccal administration. In: Bussmann WD, Dries RR, Wagner W. editors. Controlled release nitroglycerin in buccal and oral form. Basle: Karger; 1982. p. 17-25.

8. Schor JM, Davis SS, Nigalaye A, Bolton S. Susadrin transmucosal tablets. Drug Dev Ind Pharm 1983;9:1359-77.

9. Ishida M, Nambu N, Nagai T. Mucosal dosage form of lidocaine for a toothache using hydroxypropyl cellulose. Chem Pharm Bull 1982;30:980-4.

10. Bremecker KD, Strempel H, Klein G. Novel concept for a mucosal adhesive ointment. J Pharm Sci 1984;73:548-52.

11. Anders R, Merkle HP. Evaluation of laminated mucoadhesive patches for buccal drug delivery. Int J Pharm 1989;49:231-40.

12. Tanaka M, Yanagibashi N, Fukuda H, Nagai T. Absorption of salicylic acid through the oral mucous membrane of hamster cheek pouch. Chem Pharm Bull 1980;28:1056-61. 
13. Squier CA, Hall BK. The permeability of mammalian nonkeratinized oral epithelial to horseradish Peroxidase applied in vivo and in-vitro. Arch Oral Biol 1984;29:45-50.

14. Brun PPHL, Fox PLA, Vries MED, Bodde HE. In vitro penetration of some $\beta$ adrenoreceptor blocking drugs through the porcine buccal mucosa. Int J Pharm 1989;49:141-5.

15. Biswajit Basu1, Kevin Garala, Thimmasetty J. Formulation and evaluation of pimozide buccal mucoadhesive patches. Int J Pharm Sci Nanotechnol 2010;2:152.

16. Chinna Reddy P, Ramesh G, Shravan Kumar Y, Madhusudan Rao Y. Development of bioadhesive buccal tablets for felodipine and pioglitazone in combined dosage form: in vitro, ex vivo and in vivo characterization. Drug Delivery 2011;18 Suppl 5:344-52.

17. Chinna Reddy P, Madhusudan Rao Y. Buccal drug delivery systems. In: Advances in drug delivery, published by Pharma Book Syndicate. Vol. 1; 2010. p. 139-210.

18. Shayeda, Ramesh G, Chinna Reddy P, Madhusudan Rao Y. Development of novel bioadhesive buccal formulation of diltiazem: in vitro and in vivo characterization. PDA J Pharm Sci Technol 2009;63 Suppl 4:1-9.

19. Vamshi Vishnu Y, Chandra Sekhar K, Ramesh G, Madhusudan Rao Y. Development mucoadhesive patches for buccal administration of carvedilol. Curr Drug Delivery 2007;4:27-39.

20. K Chandra Sekhar, KVS Naidu, G Ramesh, Y Vamshi Vishnu, Y Madhusudan Rao. Transbuccal delivery of chlorpheniramine maleate from mucoadhesive buccal patches. Curr Drug Delivery 2008;15:185-91.

21. S Himabindu, D Sathish, Shayeda. Formulation and in vitro evaluation of mucoadhesive buccal patches of cyproheptadine hydrochloride. J Appl Pharm Sci 2012;2 Suppl 7:196-201.

22. Mona semalty, A Semalty, G Kumar. Formulation and characterization of mucoadhesive buccal films of Glipizide. Indian J Pharm Sci 2008;70 Suppl 1:43-8.
23. Amanpreet kaur, Gurpreet kaur. Mucoadhesive buccal patches based on interpolymer complexes of chitosan-pectin for delivery of Carvedilol. Saudi Pharm J 2012;20 Suppl 1:21-7.

24. Vishnu M Patel, Bhupendra G Prajapati. Design and in vitro characterization of eudragit containing mucoadhesive buccal patches. Int J PharmTech Res 2009;1 Suppl 3:783-9.

25. Bottenberg P, Cleymaet R, Muynek CD, Remon JP, Coomans D, Slop D. Development and testing of bioadhesive, fluoridecontaining slow-release tablets for oral use. J Pharm Pharmacol 1991;43:457-64.

26. Chinna Reddy Palem, Ramesh Gannu, Narender Doodipala, Vamshi Vishnu Yamsani, Madhusudan Rao Yamsani. Transmucosal delivery of domperidone from bilayered buccal patches: in vitro, ex vivo and in vivo characterization. Arch Pharm Res 2011;34 Suppl 10:1701-10.

27. Wong $\mathrm{CF}$, Yuen KH, Peh KK. An intro method for buccal adhesion studies: the importance of instrument variables. Int J Pharm 1999;180:47-57.

28. Parthasarathy Govindasamy, Bhaskar Reddy Kesavan, Jayaveera Korlakunta Narasimha. Formulation of unidirectional release buccal patches of carbamazepine and study of permeation through the porcine buccal mucosa. Asian Pac J Trop Biomed 2013;3:995-1002.

29. Peppas NA. Analysis of fickian and non-fickian drug release from polymers. Pharm Acta Helvetiae 1985;60:110-1.

30. Korsmeyer RW, Gurny R, Doelker E, Buri P, Peppas NA. Mechanism of solute release from porous hydro-matrices and other factors may be responsible. Int J Pharm 1983;15 Suppl 1:25-35.

\section{How to cite this article}

- Himabindu Peddapalli, Krishna Mohan Chinnala, Nagaraj Banala. Design and in vitro characterization of mucoadhesive buccal patches of duloxetine hydrochloride. Int J Pharm Pharm Sci 2017;9(2):52-59. 